\title{
DUAN'S FIXED POINT THEOREM: PROOF AND GENERALIZATION
}

\author{
MARTIN ARKOWITZ
}

Received 25 July 2004; Revised 6 January 2005; Accepted 21 July 2005

Let $X$ be an H-space of the homotopy type of a connected, finite CW-complex, $f: X \rightarrow X$ any map and $p_{k}: X \rightarrow X$ the $k$ th power map. Duan proved that $p_{k} f: X \rightarrow X$ has a fixed point if $k \geq 2$. We give a new, short and elementary proof of this. We then use rational homotopy to generalize to spaces $X$ whose rational cohomology is the tensor product of an exterior algebra on odd dimensional generators with the tensor product of truncated polynomial algebras on even dimensional generators. The role of the power map is played by a $\theta$-structure $\mu_{\theta}: X \rightarrow X$ as defined by Hemmi-Morisugi-Ooshima. The conclusion is that $\mu_{\theta} f$ and $f \mu_{\theta}$ each has a fixed point.

Copyright (c) 2006 Martin Arkowitz. This is an open access article distributed under the Creative Commons Attribution License, which permits unrestricted use, distribution, and reproduction in any medium, provided the original work is properly cited.

\section{Introduction}

Let $G$ be a topological group and $f: G \rightarrow G$ a map (i.e., a continuous function). Let $p_{k}: G \rightarrow G$ be the $k$ th power map defined by $p_{k}(x)=x^{k}$. Recall that a fixed point of $f$ is an element $x_{0} \in G$ such that $f\left(x_{0}\right)=x_{0}$. In 1993 Duan Haibo proved the following interesting fixed point theorem.

Theorem 1.1 [1]. If $G$ is a compact, connected topological group and $f: G \rightarrow G$ is a map, then for any $k \geq 2$, the map $p_{k} f: G \rightarrow G$ has a fixed point.

This theorem was proved more generally for homotopy-associative $\mathrm{H}$-spaces having the homotopy type of a finite, connected CW-complex (Theorem 2.2). In 1996, Lupton and Oprea [2] gave a new proof of Duan's theorem using rational homotopy theory. In 1997, Hemmi-Morisugi-Ooshima [3] extended Duan's theorem to spaces more general than homotopy-associative $\mathrm{H}$-spaces. In all of the above results, the existence of a fixed point of a map was obtained by showing the Lefschetz number of the map is non-zero.

The purpose of this paper is two-fold. First, we give a new, short proof of Duan's theorem. The proof is elementary in that the only non-trivial result required is the 
2 Duan's fixed point theorem: proof and generalization

Hopf-Leray-Samelson theorem on the rational cohomology of a homotopy-associative $\mathrm{H}$-space. Secondly, we use rational methods, in particular, a result of Halperin [4], and ideas from [3] to generalize Duan's theorem.

\section{Duan's theorem}

We begin by briefly discussing the Lefschetz number and $\mathrm{H}$-spaces. All spaces will be assumed to have the homotopy type of a finite, connected CW-complex (though this assumption can be weakened). The cohomology of a space with coefficients in the additive group of rationals will be written $H^{*}(X)=\left\{H^{n}(X)\right\}$, so that cohomology will always be taken with rational coefficients. A map $f: X \rightarrow X$ induces a linear transformation $f^{* n}: H^{n}(X) \rightarrow H^{n}(X)$. The Lefschetz number is defined by

$$
L(f)=\sum_{n=0}^{N}(-1)^{n} \operatorname{Tr}\left(f^{* n}\right),
$$

where $H^{i}(X)=0$, for $i>N$, and $\operatorname{Tr}$ denotes the trace. Lefschetz's famous fixed point theorem asserts that if $L(f) \neq 0$, then $f$ has a fixed point [5].

Next we state some basic facts about $H$-spaces. An $H$-space consists of a space $X$ and a map $m: X \times X \rightarrow X$ (called the multiplication) such that $m$ restricted to each factor is homotopic to the identity map id. For an $\mathrm{H}$-space $X$, the power map $p_{k}: X \rightarrow X, k \geq 1$, is inductively defined as follows: $p_{1}=\mathrm{id}$, and $p_{k}$ is the composition

$$
X \stackrel{\Delta}{\longrightarrow} X \times X \stackrel{p_{k-1} \times \text { id }}{\longrightarrow} X \times X \stackrel{m}{\longrightarrow} X
$$

where $\Delta$ is the diagonal map. The multiplication $m$ induces a homomorphism $m^{*}$ : $H^{*}(X) \rightarrow H^{*}(X \times X) \approx H^{*}(X) \otimes H^{*}(X)$. An element $x \in H^{n}(X)$ is called primitive if $m^{*}(x)=x \otimes 1+1 \otimes x$. If $x \in H^{n}(X)$ is primitive, then it follows immediately from the definitions that

$$
p_{k}^{*}(x)=k x
$$

The $\mathrm{H}$-space $X$ is said to be homotopy-associative if the maps $m(m \times \mathrm{id}), m(\mathrm{id} \times m)$ : $X \times X \times X \rightarrow X$ are homotopic. The Hopf-Leray-Samelson theorem ([6, page 268] and $[7$, Theorem 7.20]) asserts that if $X$ is a homotopy-associative $\mathrm{H}$-space, then

$$
H^{*}(X)=\Lambda\left(x_{1}, x_{2}, \ldots, x_{r}\right)
$$

an exterior algebra on odd degree generators $x_{1}, x_{2}, \ldots, x_{r}$ which are primitive.

With these generalities out of the way, we state an obvious lemma and proceed with Duan's theorem and its proof. 
LEMma 2.1. If $A$ is an $n \times n$ matrix of rationals and $B$ is a diagonal $n \times n$ matrix of rationals,

$$
A=\left(\begin{array}{ccc}
a_{11} & a_{12} \ldots & a_{1 n} \\
a_{21} & a_{22} \ldots & a_{2 n} \\
\vdots & \vdots & \vdots \\
a_{n 1} & a_{n 2} \ldots & a_{n n}
\end{array}\right), \quad B=\left(\begin{array}{ccc}
b_{1} & 0 \ldots & 0 \\
0 & b_{2} \ldots & 0 \\
\vdots & \vdots & \vdots \\
0 & 0 \ldots & b_{n}
\end{array}\right) \text {, }
$$

then $\operatorname{Tr}(A B)=a_{11} b_{1}+a_{22} b_{2}+\cdots+a_{n n} b_{n}=\operatorname{Tr}(B A)$.

We write the diagonal matrix $B$ as $\operatorname{diag}\left(b_{1}, b_{2}, \ldots, b_{n}\right)$.

Theorem 2.2 [1]. If $X$ is a homotopy-associative $H$-space, $f: X \rightarrow X$ any map and $p_{k}$ : $X \rightarrow X$ the kth power map, $k \geq 2$, then $p_{k} f: X \rightarrow X$ has a fixed point.

Proof. We show that $L\left(p_{k} f\right) \neq 0$. For this we consider the trace of $\left(p_{k} f\right)^{* n}=f^{* n} p_{k}^{* n}$

$$
H^{n}(X) \stackrel{p_{k}^{* n}}{\longrightarrow} H^{n}(X) \stackrel{f^{* n}}{\longrightarrow} H^{n}(X)
$$

By the theorem of Hopf-Leray-Samelson,

$$
H^{*}(X)=\Lambda\left(x_{1}, x_{2}, \ldots, x_{r}\right),
$$

where the $x_{i}$ are primitive elements of odd degree $\left|x_{i}\right|=m_{i}$. If $n \geq 1$ then a basis of $H^{n}(X)$ consists of elements

$$
y_{i_{1} i_{2} \cdots i_{l}}=x_{i_{1}} x_{i_{2}} \cdots x_{i_{l}}
$$

where $l \geq 1,1 \leq i_{1}<i_{2}<\cdots<i_{l} \leq r$ and $m_{i_{1}}+m_{i_{2}}+\cdots+m_{i_{l}}=n$. We examine the matrix of $p_{k}^{* n}$ and $f^{* n}$ with respect to this basis. Since $p_{k}^{*}\left(x_{i}\right)=k x_{i}$,

$$
p_{k}^{* n}\left(y_{i_{1} i_{2} \cdots i_{l}}\right)=k^{l} y_{i_{1} i_{2} \cdots i_{l}} \text {. }
$$

Now suppose that there are $b_{1}^{(n)}$ basis elements in $H^{n}(X)$ of length one (i.e., those of the form $\left.y_{i}\right), b_{2}^{(n)}$ basis elements in $H^{n}(X)$ of length two (i.e., those of the form $y_{i_{1} i_{2}}, i_{1}<i_{2}$ ), etc., where $b_{i}^{(n)} \geq 0$. Then the matrix $B$ of $p_{k}^{* n}$ is diagonal,

$$
B=\operatorname{diag}(\underbrace{k, \ldots, k}_{b_{1}^{(n)}}, \underbrace{k^{2}, \ldots, k^{2}}_{b_{2}^{(n)}}, \ldots) .
$$

Next we consider the matrix $A$ of $f^{* n}$ with respect to this basis. Now $f^{* n}$ is obtained by taking the homomorphism on integral $n$-dimensional cohomology induced by $f$ and tensoring it with the rationals. Thus $A$ is a matrix of integers. Let $e_{1}^{(n)}$ be the sum of the first $b_{1}^{(n)}$ diagonal entries of $A, e_{2}^{(n)}$ the sum of the next $b_{2}^{(n)}$ diagonal entries, etc. Then by Lemma 2.1,

$$
\operatorname{Tr}\left(\left(p_{k} f\right)^{* n}\right)=\operatorname{Tr}(A B)=k e_{1}^{(n)}+k^{2} e_{2}^{(n)}+\cdots+k^{r} e_{r}^{(n)}
$$


Thus $\operatorname{Tr}\left(\left(p_{k} f\right)^{* n}\right) \equiv 0(\bmod k)$ for $n \geq 1$, and so $L\left(p_{k} f\right) \equiv 1(\bmod k)$. Since $k \geq 2$, we have $L\left(p_{k} f\right) \neq 0$. This completes the proof.

Remark 2.3. There are some simple extensions of Theorem 2.2:

(1) If the H-space $X$ has a homotopy inverse, one can define $p_{k}$ for all integers $k$. Theorem 2.2 then holds for all $|k| \geq 2$.

(2) Theorem 2.2 holds for the map $f p_{k}: X \rightarrow X$ since it is well known that $L\left(f p_{k}\right)=$ $L\left(p_{k} f\right)$.

(3) Let $k$ be an integer such that $|k| \geq 2$. Suppose that $X$ is a homotopy-associative $\mathrm{H}$ space and there is a map $\mu: X \rightarrow X$ such that $\mu^{*}\left(x_{i}\right)=a_{i} x_{i}$, where $a_{i} \equiv 0(\bmod k)$, for $i=1,2, \ldots, r$. Then the previous proof shows that if $f: X \rightarrow X$ is any map, then $\mu f$ and $f \mu$ each has a fixed point. We will return to this in Section 4.

We note the following immediate consequence of Duan's theorem which appears in [5, Theorem 1, page 49].

Corollary 2.4. Let $G$ be a compact, connected topological group, $a \in G$ and $k \geq 2$. Then there exists $x_{0} \in G$ such that $x_{0}^{k}=a$.

Proof. Let $L_{a}: G \rightarrow G$ be left multiplication by $a$. By Duan's theorem, $L_{a^{-1}} p_{k+1}$ has a fixed point $x_{0}$.

\section{Fixed points and eigenvalues}

In this section we consider spaces with restricted cohomology and state a result on the Lefschetz number of self maps of such spaces. This result, Theorem 3.1, which may be of some interest in itself, will be used to generalize Duan's theorem in Section 4.

Let $Y$ be a space and consider the vector space $I^{*}\left(H^{*}(Y)\right)=\left\{I^{n}\left(H^{*}(Y)\right)\right\}$ of indecomposables of $H^{*}(Y)$ defined by

$$
I^{*}\left(H^{*}(Y)\right)=\frac{H^{+}(Y)}{H^{+}(Y) \cdot H^{+}(Y)},
$$

where $H^{+}$denotes positive-dimensional cohomology. A map $f: Y \rightarrow Y^{\prime}$ induces $f^{*}$ : $H^{*}\left(Y^{\prime}\right) \rightarrow H^{*}(Y)$ and this induces a linear transformation $I^{*}\left(f^{*}\right): I^{*}\left(H^{*}\left(Y^{\prime}\right)\right) \rightarrow$ $I^{*}\left(H^{*}(Y)\right)$.

For the rest of this section we consider spaces $X$ whose cohomology has the following form

$$
H^{*}(X)=\Lambda\left(x_{1}, x_{2}, \ldots, x_{r}\right) \otimes P\left(y_{1}, y_{2}, \ldots, y_{s}\right) /\left\langle y_{1}^{n_{1}}, y_{2}^{n_{2}}, \ldots, y_{s}^{n_{s}}\right\rangle
$$

where $\Lambda\left(x_{1}, x_{2}, \ldots, x_{r}\right)$ is an exterior algebra on odd dimensional generators $x_{1}, x_{2}, \ldots, x_{r}$, $P\left(y_{1}, y_{2}, \ldots, y_{s}\right)$ is a polynomial algebra on even dimensional generators $y_{1}, y_{2}, \ldots, y_{s}$ and $\left\langle y_{1}^{n_{1}}, y_{2}^{n_{2}}, \ldots, y_{s}^{n_{s}}\right\rangle$ is the ideal generated by the powers $y_{1}^{n_{1}}, y_{2}^{n_{2}}, \ldots, y_{s}^{n_{s}}$. In short, $H^{*}(X)$ is a tensor product of monogenic algebras.

We will always assume for a space $X$ which satisfies (3.2) that $1<n_{1}<n_{2}<\cdots<n_{s}$.

We give examples of such spaces in Examples 3.3(1). 
Now let $X$ be a space satisfying (3.2) and $f: X \rightarrow X$ a map. The vector space of indecomposables $I^{*}\left(H^{*}(X)\right)$ can be split into its odd and even degree parts

$$
I^{*}\left(H^{*}(X)\right)=V \oplus W
$$

where $V=\bigoplus_{\text {iodd }} I^{i}\left(H^{*}(X)\right)$ and $W=\bigoplus_{i \text { even }} I^{i}\left(H^{*}(X)\right)$. Then $I^{*}\left(f^{*}\right): I^{*}\left(H^{*}(X)\right) \rightarrow$ $I^{*}\left(H^{*}(X)\right)$ induces linear transformations

$$
f_{V}: V \longrightarrow V, \quad f_{W}: W \longrightarrow W
$$

The following theorem will be proved in Section 5 .

Theorem 3.1. Let $X$ be a space satisfying (3.2) and $f: X \rightarrow X$ a map. Suppose that -1 is not an eigenvalue of $f_{W}$. Then

$$
L(f) \neq 0 \Longleftrightarrow f_{V} \text { has no eigenvalue equal to } 1 \text {. }
$$

We make some remarks on the theorem.

Remarks 3.2. (1) The matrices of the linear transformations $f_{V}$ and $f_{W}$ can be determined from the induced linear transformation $f^{*}$ applied to the algebra generators $x_{1}, x_{2}, \ldots$, $x_{r}, y_{1}, y_{2}, \ldots, y_{s}$ of $H^{*}(X)$. In general, it is difficult to calculate the eigenvalues of a linear transformation since this requires finding the roots of the characteristic polynomial. In applying Theorem 3.1 to show $L(f) \neq 0$, however, it is only necessary to show that -1 and 1 are not roots of the appropriate characteristic polynomials. This is much easier to do.

(2) The theorem holds if $r$ or $s=0$. In addition, the conclusion $L(f) \neq 0$ holds without the hypothesis that $f_{V}$ has no eigenvalue equal to 1 , provided all $n_{i}$ are odd. This can be seen from the proof.

(3) A result similar to Theorem 3.1 has been proved by Lupton and Oprea [2]. In Remark 5.3 we discuss the relation of their result to our work.

We next give some examples related to Theorem 3.1.

Examples 3.3. (1) We indicate one way (though not the only way) to construct spaces $X$ satisfying (3.2). Let $A$ be a space such that $H^{*}(A)=\Lambda\left(x_{1}, \ldots, x_{r}\right)$. For example, $A$ could be the product of any number of the following spaces: homotopy-associative $\mathrm{H}$-spaces and odd dimensional spheres. Let $B$ be a space such that $H^{*}(B)=P\left(y_{1}, \ldots, y_{s}\right) /\left\langle y_{1}^{n_{1}}, \ldots, y_{s}^{n_{s}}\right\rangle$. For example, $B$ could be the product of any number of the following spaces: projective spaces and even dimensional spheres. Then $X=A \times B$ is a space which satisfies (3.2).

(2) We next show that the hypothesis that -1 is not an eigenvalue of $f_{W}$ is necessary in general. Let $X$ be the complex projective space $\mathbb{C} P^{2 n+1}$ and let $f: X \rightarrow X$ be a map of degree -1 , that is, $f^{* 2}(u)=-u$ for every $u \in H^{2}(X)$. Then 1 is not an eigenvalue of $f_{V}$, -1 is an eigenvalue of $f_{W}$ and $L(f)=0$.

(3) Here we show that the strict inequality $1<n_{1}<n_{2}<\cdots<n_{r}$ is necessary in Theorem 3.1. Define $f: S^{2} \times S^{2} \rightarrow S^{2} \times S^{2}$ by $f(x, y)=(y,-x)$. Let $\{u, v\} \subseteq H^{2}\left(S^{2} \times S^{2}\right)$ be the basis corresponding to the two 2-spheres. Then $f^{* 2}(u)=v, f^{* 2}(v)=-u$ and $f^{* 4}(u v)=-u v$, and so $L(f)=0$. 


\section{Theta spaces}

In this section we will use Theorem 3.1 to extend Duan's theorem to spaces $X$ which satisfy (3.2). In order to do this it is necessary to describe a map $X \rightarrow X$ which plays the role of the power map of H-spaces. This has been done by Hemmi-Morisugi-Ooshima [3]. We begin this section by summarizing their work (with some small changes in terminology).

For the remainder of the paper we will use $X$ to denote a space which satisfies (3.2) of Section 3 and will use $Y$ to denote an arbitrary space (of the homotopy type of a finite, connected CW-complex).

Definition 4.1. Let $Y$ be a space and $\left\{m_{1}, m_{2}, \ldots, m_{t}\right\}$ a set of positive integers defined as follows:

$$
I^{m}\left(H^{*}(Y)\right) \neq 0 \Longleftrightarrow m=m_{i}, \quad \text { for some } i=1,2, \ldots, t \text {. }
$$

Let $\theta:\left\{m_{1}, m_{2}, \ldots, m_{t}\right\} \rightarrow \mathbb{Z}$ be an integer-valued function. Then a $\theta$-structure on $Y$ is a map $\mu_{\theta}: Y \rightarrow Y$ such that

$$
I^{m_{i}}\left(\mu_{\theta}^{*}\right)(y)=\theta\left(m_{i}\right)(y),
$$

for every $y \in I^{m_{i}}\left(H^{*}(Y)\right)$. The pair $\left(Y, \mu_{\theta}\right)$ (or just $\left.Y\right)$ is called a $\theta$-space. A constant $\theta$ structure is one such that $\theta\left(m_{i}\right)=k$, for all $i$, where $k \in \mathbb{Z}$ is a fixed integer.

There is a long list of $\theta$-spaces in [3] and we mention some of them below. All $\theta$ functions in the following list have the form $\theta\left(m_{i}\right)=k^{e\left(m_{i}\right)}$, for some integer $k$ and function $e$.

(i) $\mathrm{H}$-spaces and co-H-spaces have constant $\theta$-structure given by the power map.

(ii) Semi-simple Lie groups $G$ and their classifying spaces $B G$ have $\theta$-structure given by the unstable Adams operations $\psi^{k}$ on $B G$ and $\Omega \psi^{k}$ on $\Omega B G=G$, for certain $k$.

(iii) Complex and Quaternionic Grassman manifolds $G_{p, q}$ with some restrictions on $p$ and $q$ have $\theta$-structure.

(iv) The Stiefel manifolds $U(2 n+2) / U(2 n)$ have constant $\theta$-structure $k$ if and only if $k \equiv 0,1,5(\bmod 8)$.

In addition, the existence of $\theta$-structure on a large class of spaces is obtained from the following corollary of Theorem 1 in [3]:

If $X$ is a simply-connected space which satisfies (3.2) of Section 3, then there exists infinitely many $\theta$-structures on $X$.

In Theorem 2 of [3] the authors consider self maps $f: Y \rightarrow Y$ of a $\theta$-space, for certain $\theta$, and show the existence of fixed points of $f \mu_{\theta}$ and $\mu_{\theta} f$. The restrictions on $\theta$ are that $\theta\left(m_{i}\right)=k^{e\left(m_{i}\right)}$, where $e\left(m_{i}\right)=(b-a) m_{i}+2 a-b$, for $b \geq a \geq 1$ and $|k| \geq 2$. We prove a similar theorem below (by different methods) which restricts the spaces to those satisfying (3.2) but allows a much larger class of functions $\theta$.

Theorem 4.2. Let $X$ be any space satisfying (3.2) and $f: X \rightarrow X$ any map. Let $\left\{m_{1}, m_{2}, \ldots, m_{t}\right\}$ be the set of degrees of the non-zero indecomposables of $H^{*}(X)$ and let $\theta:\left\{m_{1}, m_{2}, \ldots, m_{t}\right\} \rightarrow \mathbb{Z}-\{0, \pm 1\}$ be any function. If $\mu_{\theta}: X \rightarrow X$ is a $\theta$-structure on $X$, then $f \mu_{\theta}$ and $\mu_{\theta} f$ each has a fixed point. 
Proof. We apply Theorem 3.1 to $\mu_{\theta} f: X \rightarrow X$. We decompose $I^{*}\left(H^{*}(X)\right)=V \oplus W$ into odd and even parts and first consider $\left.I^{*}\left(f^{*} \mu_{\theta}^{*}\right)\right|_{W}=\left(\mu_{\theta} f\right)_{W}: W \rightarrow W$. Suppose $w \in W^{m_{i}}$ is an eigenvector of $\left(\mu_{\theta} f\right)_{W}$ with eigenvalue -1 . Then

$$
f_{W}\left(\theta\left(m_{i}\right)(w)\right)=I^{m_{i}}\left(f^{*} \mu_{\theta}^{*}\right)(w)=\left(\mu_{\theta} f\right)_{W}(w)=-w
$$

and so $f_{W}(w)=\left(-1 / \theta\left(m_{i}\right)\right) w$. Thus $-1 / \theta\left(m_{i}\right)$ is an eigenvalue of $f_{W}$ which is a rational number. But $f_{W}$ is induced by a map $f: X \rightarrow X$ and so, as noted in the proof of Theorem 2.2, with respect to some basis of $W, f_{W}$ is represented by an integral matrix (see also $[2, \S 3]$ ). But the only rational eigenvalues of an integral matrix are integers $[8$, Theorem 4.16]. Therefore $-1 / \theta\left(m_{i}\right)$ cannot be an eigenvalue of $f_{W}$ since $\theta\left(m_{i}\right) \neq \pm 1$. Thus -1 is not an eigenvalue of $f_{W}$. A similar argument shows that 1 is not an eigenvalue of $f_{V}$. Therefore by Theorem 3.1,L( $\left.\mu_{\theta} f\right) \neq 0$. An analogous argument holds for $f \mu_{\theta}$.

Remark 4.3. We illustrate how Theorem 4.2 can be used in some concrete examples. Let $X=A \times B$ be a space of the type discussed in Examples 3.3(1). If $\mu_{\theta}$ is a $\theta$-structure on $A$ and $\mu_{\theta^{\prime}}$ is a $\theta$-structure on $B$, then $\mu_{\theta} \times \mu_{\theta^{\prime}}$ is a $\theta$-structure on $A \times B$. More specifically, suppose $A$ is a homotopy-associative $H$-space with $\mu_{\theta}$ the $k$ th power map and $B$ is a product of even dimensional spheres and projective spaces with $\mu_{\theta^{\prime}}$ a $\theta$-structure which is constant at $l$ (for example, the product of maps of degree $l$ ). If $k$ and $l$ are both $\neq 0, \pm 1$, then Theorem 4.2 applies to the $\theta$-structure $\mu_{\theta} \times \mu_{\theta^{\prime}}$ on $X$.

\section{Proof of Theorem 3.1}

We state a special case of a theorem of Halperin which will be needed to prove Theorem 3.1. This requires the use of rational homotopy theory, in particular, Sullivan minimal models (see [9] and [10]). For a space $X$ which satisfies (3.2), one can construct the minimal model $\mathcal{M}$ of $X$. This has the following properties: $\mathcal{M}$ is a free-commutative, graded, differential algebra with generators $x_{1}, \ldots, x_{r}, y_{1}, \ldots, y_{s}$ (which are in one-one correspondence with the generators of $H^{*}(X)$ and have the same degree) and generators $z_{1}, \ldots, z_{s}$ with $\left|z_{i}\right|=\left|y_{i}\right| n_{i}-1$. Then

$$
M=\Lambda\left(x_{1}, \ldots, x_{r}, z_{1}, \ldots, z_{s}\right) \otimes P\left(y_{1}, \ldots, y_{s}\right),
$$

with $\left|x_{i}\right|$ and $\left|z_{i}\right|$ odd and $\left|y_{i}\right|$ even. Note that a vector space basis for $M$ consists of all $x_{1}^{\eta_{1}} \cdots x_{r}^{\eta_{r}} y_{1}^{\lambda_{1}} \cdots y_{s}^{\lambda_{s}} z_{1}^{\tau_{1}} \cdots z_{s}^{\tau_{s}}$, where $0 \leq \eta_{i}, \tau_{i} \leq 1$ and $0 \leq \lambda_{i}$. The differential $d$ on $\mathcal{M}$ is defined by: $d x_{i}=0, d y_{i}=0$ and $d z_{i}=y_{i}^{n_{i}}$. Clearly $H^{*}(\mathcal{M}, d)=H^{*}(X)$. We split the vector space $I^{*}(\mathcal{M})$ of indecomposables of $\mathcal{M}$ into the direct sum of an odd degree part $O$ and an even degree part $E$. We identify $O=\left\langle x_{1}, \ldots, x_{r}, z_{1}, \ldots, z_{s}\right\rangle$ and $E=\left\langle y_{1}, \ldots, y_{s}\right\rangle$. A map $f$ : $X \rightarrow X$ induces a homomorphism $\phi: \mathcal{M} \rightarrow \mathcal{M}$. This determines $I^{*}(\phi): I^{*}(\mathcal{M}) \rightarrow I^{*}(\mathcal{M})$ and thence $\phi_{O}: O \rightarrow O$ and $\phi_{E}: E \rightarrow E$. We now state a special case of Halperin's theorem for spaces which satisfy (3.2).

Theorem 5.1 [4, Theorem 3]. The number of eigenvalues of $\phi_{O}$ which are 1 equals the number of eigenvalues of $\phi_{E}$ which are 1 if and only if $L(f) \neq 0$. 
8 Duan's fixed point theorem: proof and generalization

Using this theorem, we now prove Theorem 3.1.

Proof. We fix $l, 1 \leq l \leq s$, and write $\phi\left(z_{l}\right)$ as a linear combination of basis elements in the vector space $M$,

$$
\phi\left(z_{l}\right)=\sum_{i} a_{l i} z_{i}+\sum_{j} b_{l j} x_{j}+\epsilon_{l}
$$

where $\epsilon_{l}$ is decomposable and $\left|z_{l}\right|=\left|z_{i}\right|=\left|x_{j}\right|$ for all $i$ and $j$ in the above sums. We let $I=\left\{i|| z_{i}|=| z_{l} \mid\right\}$ and apply $d$ to both sides of (5.2) to obtain

$$
\phi\left(d z_{l}\right)=\sum_{i \in I} a_{l i} y_{i}^{n_{i}}+d \epsilon_{l}
$$

But $\phi\left(d z_{l}\right)=\phi\left(y_{l}^{n_{l}}\right)=\left(\phi\left(y_{l}\right)\right)^{n_{l}}$, and so (5.3) yields

$$
\left(\phi\left(y_{l}\right)\right)^{n_{l}}=\sum_{i \in I} a_{l i} y_{i}^{n_{i}}+d \epsilon_{l}
$$

Next we write $\phi\left(y_{l}\right)$ as a linear combination of basis elements

$$
\phi\left(y_{l}\right)=\sum_{k \in K} c_{l k} y_{k}+\delta_{l},
$$

where $K=\left\{k|| y_{k}|=| y_{l} \mid\right\}$ and $\delta_{l}$ is decomposable. Since $n_{1}<\cdots<n_{s}$, it follows that $I \cap K=\{l\}$. Then we obtain from (5.4) and (5.5),

$$
\left(\sum_{k \in K} c_{l k} y_{k}+\delta_{l}\right)^{n_{l}}=\sum_{i \in I} a_{l i} y_{i}^{n_{i}}+d \epsilon_{l}
$$

Consider any $t$ with $1 \leq t \leq s$. We will equate the terms which are linear combinations of $y_{t}^{a}$ for all $a>0$ on the left side of (5.6) with those on the right side of (5.6). For this it is necessary to analyze the elements $d \epsilon_{l}$ and $\delta_{l}$ in terms of the vector space basis above, noting that $\epsilon_{l}$ and $\delta_{l}$ are decomposable and that $\left|d \epsilon_{l}\right|=\left|z_{l}\right|+1$ and $\left|\delta_{l}\right|=\left|y_{l}\right|$. Now $\delta_{l}$ may contain a term of the form $u_{a} y_{t}^{a}$, where $u_{a}$ is a rational and $a \geq 2$. Thus the only possible terms on the left side of (5.6) which are powers of $y_{t}$ are $c_{l t}^{n_{l}} y_{t}^{n_{l}}$ and $u_{a}^{n_{l}} y_{t}^{a n_{l}}$. For the right side of (5.6) note that $\epsilon_{l}$ may contain a term of the form $v_{b} y_{t}^{b} z_{t}$, where $b>0$ and $v_{b}$ is a rational. Then $d \epsilon_{l}$ will contain $v_{b} y_{t}^{n_{t}+b}$. Thus the only possible terms which are powers of $y_{t}$ on the right side of (5.6) are $a_{l t} y_{t}^{n_{t}}$ and $v_{b} y_{t}^{n_{t}+b}$. Now suppose $t \in K$ and $t \neq l$. Then $t \notin I$ and (5.6) yields

$$
c_{l t}^{n_{l}} y_{t}^{n_{l}}=v_{b} y_{t}^{n_{t}+b}
$$

Thus if $t>l$, then $n_{l}<n_{t}$, and so $c_{l t}=0$. Next suppose $t \in I$ and $t \neq l$. Then $t \notin K$ and (5.6) yields

$$
u_{a}^{n_{l}} y_{t}^{a n_{l}}=a_{l t} y_{t}^{n_{t}}
$$


If $l>t$, then $a n_{l}>n_{t}$, and so $a_{l t}=0$. Finally, if $l=t$, then $c_{l l}^{n_{l}}=a_{l l}$. Putting this information into (5.2) and (5.5), we have

$$
\begin{gathered}
\phi_{O}\left(z_{l}\right)=\sum_{i \geq l} a_{l i} z_{i}+\sum_{j} b_{l j} x_{j}, \\
\phi_{E}\left(y_{l}\right)=\sum_{k \leq l} c_{l k} y_{k},
\end{gathered}
$$

where $a_{l l}=c_{l l}^{n_{l}}$. Now $E=\left\langle y_{1}, \ldots, y_{s}\right\rangle$ and $\phi_{E}: E \rightarrow E$. From (5.10), the eigenvalues of $\phi_{E}$ (in degree $\left|y_{l}\right|$ ) are rational numbers of the form $c_{i i}$. These are the same as the eigenvalues of $f_{W}$. By hypothesis, none of these eigenvalues equals -1 . Clearly $I^{*}(\phi):\left\langle x_{1}, \ldots, x_{r}\right\rangle \rightarrow$ $\left\langle x_{1}, \ldots, x_{r}\right\rangle$. By examining the matrix of $\phi_{O}$, we see that the eigenvalues of $\phi_{O}$ consist of those of the form $a_{i i}=c_{i i}^{n_{i}}$ together with the eigenvalues of $\phi_{O} \mid\left\langle x_{1}, \ldots, x_{r}\right\rangle$. Now $a_{i i}=1$ if and only if $c_{i i}=1$. Thus the number of eigenvalues of $\phi_{O}$ which are 1 equals the number of eigenvalues of $\phi_{E}$ which are 1 plus the number of eigenvalues of $\phi_{O} \mid\left\langle x_{1}, \ldots, x_{r}\right\rangle$ which are 1. But the eigenvalues of $\phi_{O} \mid\left\langle x_{1}, \ldots, x_{r}\right\rangle$ are just the eigenvalues of $f_{V}$. By Halperin's theorem, $L(f) \neq 0$ if and only if no eigenvalue of $f_{V}$ equals 1 .

Remark 5.2. Halperin's theorem as stated and proved in [4] is more general in two distinct ways than what we have stated above. First of all, the theorem applies to elliptic spaces. These are spaces whose (rational) cohomology and rational homotopy groups (i.e., homotopy groups tensored with the rationals) vanish in all sufficiently high dimensions. The spaces which satisfy (3.2) are elliptic spaces. Secondly, the theorem gives a formula for the Lefschetz number of a map $f$ in terms of the eigenvalues of $f_{V}$ and $f_{W}$. Since we are interested in fixed points of maps, we have only considered the case where $L(f) \neq 0$. This has led to a simplified statement of the theorem.

Remark 5.3. Lupton and Oprea consider an elliptic space $X$ whose minimal model $M$ is oddly graded, that is, $\mathcal{M}=\Lambda\left(x_{1}, \ldots, x_{r}\right)$, an exterior algebra on odd dimensional generators. It is not assumed that the differential $d=0$. If $f: X \rightarrow X$ is a map such that the induced map $\phi_{O}=I^{*}(\phi): I^{*}(\mathcal{M}) \rightarrow I^{*}(\mathcal{M})$ does not have 1 as an eigenvalue, then the main result of $[2, \S 5]$ asserts that $L(f) \neq 0$. It is possible to modify the statement and proof of Theorem 3.1 slightly so as to include this result. One assumes that the minimal model $\mathcal{M}$ of $X$ has the form

$$
M=\Lambda\left(x_{1}, \ldots, x_{r}, z_{1}, \ldots, z_{s}\right) \otimes P\left(y_{1}, \ldots, y_{s}\right),
$$

with $\left|x_{i}\right|$ and $\left|z_{i}\right|$ odd and $\left|y_{i}\right|$ even. Furthermore, $d x_{i} \in \Lambda\left(x_{1}, \ldots, x_{r}\right), d z_{i}=y^{n_{i}}$ and $d y_{i}=$ 0 with $n_{1}<\cdots<n_{s}$. We assume that $\phi_{E}: E \rightarrow E$ does not have -1 as an eigenvalue. Then the modified version of Theorem 3.1 asserts that $L(f) \neq 0$ if and only if $\phi_{O}$ does not have 1 as an eigenvalue. For the proof it is only necessary to show that $\phi_{O}\left(x_{i}\right) \in\left\langle x_{1}, \ldots, x_{r}\right\rangle$ which requires straightforward arguments similar to those given in the proof of Theorem 3.1.

\section{Acknowledgment}

We would like to thank Robert Brown, Gregory Lupton, Donald Stanley, and Peter Wong for several helpful comments. 
10 Duan's fixed point theorem: proof and generalization

\section{References}

[1] D. Haibao, A characteristic polynomial for self-maps of $H$-spaces, The Quarterly Journal of Mathematics. Oxford. Second Series (2) 44 (1993), no. 175, 315-325.

[2] G. Lupton and J. Oprea, Fixed points and powers of self-maps of $H$-spaces, Proceedings of the American Mathematical Society 124 (1996), no. 10, 3235-3239.

[3] Y. Hemmi, K. Morisugi, and H. Ooshima, Self maps of spaces, Journal of the Mathematical Society of Japan 49 (1997), no. 3, 438-453.

[4] S. Halperin, Spaces whose rational homology and de Rham homotopy are both finite-dimensional, Algebraic Homotopy and Local Algebra (Luminy, 1982), Astérisque, vol. 113-114, Soc. Math. France, Paris, 1984, pp. 198-205.

[5] R. F. Brown, The Lefschetz Fixed Point Theorem, Scott, Foresman, Illinois, 1971.

[6] E. H. Spanier, Algebraic Topology, McGraw-Hill, New York, 1966.

[7] J. W. Milnor and J. C. Moore, On the structure of Hopf algebras, Annals of Mathematics. Second Series (2) 81 (1965), 211-264.

[8] T. Hungerford, Abstract Algebra: An Introduction, Saunders college, Pennsylvania, 1990.

[9] P. A. Griffiths and J. W. Morgan, Rational Homotopy Theory and Differential Forms, Progress in Mathematics, vol. 16, Birkhäuser, Massachusetts, 1981.

[10] Y. Félix, S. Halperin, and J.-C. Thomas, Rational Homotopy Theory, Graduate Texts in Mathematics, vol. 205, Springer, New York, 2001.

Martin Arkowitz: Department of Mathematics, Dartmouth College, Hanover, NH 03755, USA

E-mail address: martin.arkowitz@dartmouth.edu 